\title{
MIXING ANISOTROPIC FORMULATION FOR ANALYSIS OF COMPOSITES
}

\author{
S. OLLER, E. OÑATE AND J. MIQUEL \\ Universitat Politècnica de Catalunya, Gran Capitán S/N, 08034 Barcelona, Spain
}

\begin{abstract}
SUMMARY
A general constitutive model adequate for analysis of the thermomechanical response of composite materials is presented. The model is based on the mixture of the basic substances of the composite and allows the evaluation of the interdependence between the constitutive behaviour of different compounding materials. The behaviour of the each compound is modelled by a general anisotropic thermo-elasto-plastic model, termed the 'base model'. The different base models for each compound are combined using mixing theory to simulate the behaviour of the multiphase material.
\end{abstract}

KEY WORDS numerical methods; constitutive equations; composite; mixing formulation; anisotropic elastoplastic model; mapping stress tensor space

\section{INTRODUCTION}

Mixing theory is based on the principle of interaction of the compounding substances and uses the following assumptions: $(a)$ each infinitesimal volume of a composite is filled by a finite number of compounding substances; $(b)$ each compounding substance participates in the behaviour of the total composite material in the same volume proportion; (c) all compounding substances have the same strains (compatibility concept); and $(d)$ the volume occupied by each compounding substance is much smaller than the total volume of the composite. Assumption (b) implies a homogeneous distribution of all substances in a certain region of the composite. The interaction between the different compounding substances, each defined by an appropriate constitutive law, yields the overall constitutive behaviour of the composite in terms of the percentage volume occupied by each substance and its distribution within the composite.

The basis of mixing theory was first derived by Trusdell and Toupin ${ }^{1}(1960)$. In this paper mixing theory is extended and used to propose an implicit non-linear constitutive model for ' $n$ phase' composite materials.

Each phase can have an anisotropic or isotropic behaviour. This possibility is defined by means of an equivalent isotropic solid as first introduced by Betten using the concept of mapped stress tensor. ${ }^{2.3}$ This offers the possibility of using all the advantages of the well known isotropic models.

The authors have developed a generalization of standard isotropic plasticity theory for the analysis of anisotropic solids in previous works. ${ }^{4.5}$ The basic idea was to model the behaviour of an anisotropic solid by means of a fictitious isotropic solid. This situation introduces a limitation

CCC 1069-8299/96/080471-12 
in the anisotropic mapped theory because it involves a proportionality concept between the yield strength and the elasticity modulus for each material direction. In the present work a generalization of such a basic theory is introduced taking into account a more general concept of mapping of stress and strain tensors ${ }^{4}$. The anisotropic behaviour is formulated by means of fictitious isotropic tensors which result from a fourth order tensor transformation of the real stress and strain field to the homonymous stress and strain spaces. This allows us to use the same yield and potential functions derived for standard isotropic materials, whereas all the relevant information on the material anisotropy properties is embedded in the fourth order transformation tensors only. The formulation presented is completely general and it allows us to model a different class of orthotropic and anisotropic materials typical in composites. The model seems to be particularly suited to be applied for analysis of multiphase materials such as fibre reinforced composites and concrete as shown in the examples presented.

\section{MIXING FORMULATION}

Mixing theory was derived to deal with the interaction of compounding substances ${ }^{1}$ of a multiphase material using local continum mechanics. This theory allows to consider the simultaneous combination of the different constitutive behaviour of each substance (i.e. elastic, elasto-plastic, elasto-brittle elasto-damage, etc.). In this paper each compounding substance behaves precisely as the thermo-anisotropic elasto-plastic material that will be described in next Section.

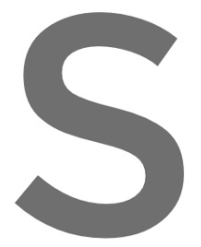

The simplest closin compounding compati moderate), therefore the

The free energy in composite butk materials can be expressed as
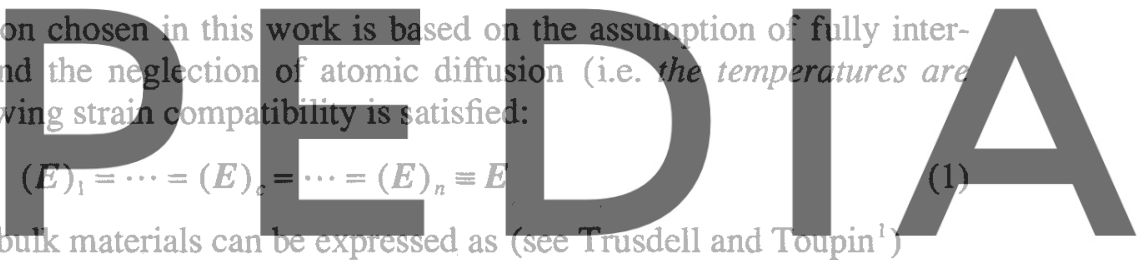

Register for free at https//www.scipedia, com to dow

$$
=\sum_{c=1}^{n} k_{c} \Psi_{c}\left(\bar{E} ; \theta ;(p)_{c}\right)
$$

where $\Psi_{c}\left(E ; \theta ;(p)_{c}\right)$ is the free energy corresponding to each of the $n$ compounding substances involved in the mixture, $k_{c}$ and $(p)_{c}$ are the volume fraction and internal variables for the ' $c$ th' substance, and $\theta$ is the free thermal variable.

Following an identical procedure as for single-phase materials, ${ }^{6,7}$ the expression of the secant constitutive equation of each phase material is obtained from the dissipation ${ }^{5,8,9}$ occurring in the real anisotropic composite material:

$$
\begin{gathered}
S_{c}=m_{c}^{o} \frac{\partial \Psi_{c}\left(E_{c}^{e} ; \theta ; \alpha_{c}^{m}\right)}{\partial E_{c}^{e}}=\mathscr{C}_{c}(\theta): \boldsymbol{E}_{c}^{e} \\
S_{c}=\mathscr{C}_{c}(\theta):\left\{\mathscr{C}^{-1}(\theta):\left[\sum_{c=1}^{n} k_{c} \mathscr{C}_{c}(\theta): E\right]-E_{c}^{p}-\overline{\boldsymbol{a}}_{c}^{\theta}\left(\theta-\theta_{o}\right)\right\}
\end{gathered}
$$

where $\boldsymbol{a}_{c}^{\theta}=\partial \boldsymbol{E}_{c}^{\theta} / \partial \theta$ is the thermal expansion coefficient for the ' $c$ th' compounding material, $\boldsymbol{E}_{c}^{\theta}$ is the thermal strain and $\theta_{o}$ is the room temperature. Equations (3) give the stress distribution for each compounding substance of the multiphase composite. 


\section{THE PLASTIC CONSTITUTIVE LAW FOR A 'BASE COMPOUNDING ANISOTROPIC MATERIAL'}

The base plastic constitutive model for each compounding material is formulated in the referential configuration using total Lagrangean kinematics, 4,5 and is included in the framework of the mixing theory previously described. The single model can deal with non-linear problems involving temperature, large plastic strains and small elastic strains. ${ }^{4}$ Obviously, simpler orthotropic and isotropic plasticity models are readily obtained as particular cases of the proposed model.

Traditional procedures for deriving the constitutive equations for anisotropic elasto-plastic materials are based on appropriate yield and potential functions described in terms of the characteristic material properties. ${ }^{4}$ Satisfaction of the invariance condition in these cases is difficult and not always possible. A procedure proposed in this work to guarantee this condition is to define the properties of the real anisotropic solid in terms of a fictitious isotropic solid (see Figure 1).

This is achieved by relating the stresses between the real and fictitious spaces using the following linear transformation:

$$
\tau_{c}=\mathscr{T}_{c}: S_{c}
$$

where $S_{c}$ and $\tau_{c}$ are the stress tensor in the 'cth' component in the real anisotropic solid and the fictitious isotropic solid, respectively, and $\sigma_{c}=f_{c}^{\tau} \otimes f_{c}^{-1}$ is a fourth order material tensor, ${ }^{4}$ termed a space transformation tensor, which depends on the strength elastic limit tensofs corresponding to the real $f_{c}$ and fictitious isotropic compounding

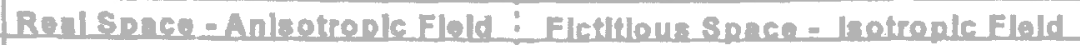

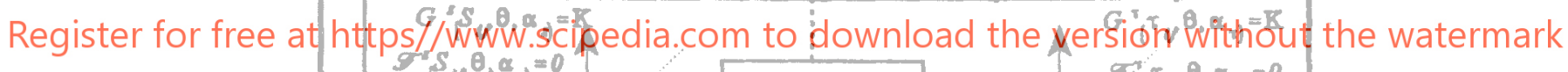

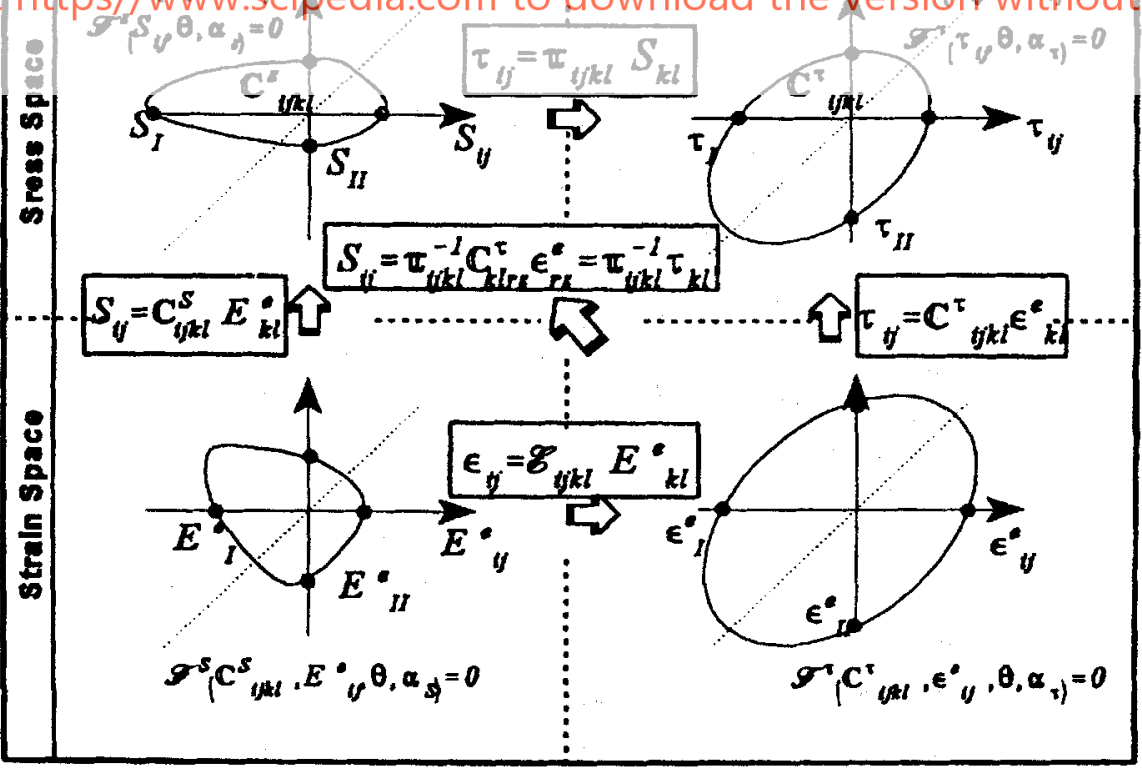

Figure 1. Relationship between 'real-fictitious' space and 'stress-strain' field 
$c$ denote from now onwards variables in the fictitious isotropic space and corresponding to the ' $c$ th' component. The mapping expressed by means of equation (4) produces a change of shape in the yield function as shown in Figure (2).

To ensure no proportionality between the strength and the elastic modulus in each particular direction, the following relation between the free mechanical variable, i.e. the real elastic strains $E_{c}^{e}$ and the fictitious ones $\varepsilon_{c}^{e}$ is defined as

$$
\varepsilon_{c}^{e}=\mathscr{E}_{c}: E_{c}^{e}
$$

This assumption implies non-uniqueness of the elastic strains when the change of space is produced. In equation (5), $\mathscr{C}_{c}=\mathscr{C}_{c}^{\tau-1}: \mathscr{T}_{c}: \mathscr{C}_{c}$ is a fourth order material tensor, termed strain space transformation tensor, ${ }^{4}$ which depends on the stress space transformation tensor $\mathscr{F}_{c}$ and also on tensors $\mathscr{C}_{c}$ and $\mathscr{C}_{c}^{\tau}$, these being the constitutive tensors in the real and fictitious spaces for the 'cth' component, respectively.

Assuming that the information concerning the material anisotropy properties is contained in tensor $\mathscr{T}_{c}$, the yield and plastic potential function for the anisotropic solid are defined as: ${ }^{4}$

$$
\begin{aligned}
& \mathscr{F}_{c}\left(S_{c} ; \theta ; \alpha_{c}^{m}\right)=\mathscr{F}_{c}^{\tau}\left(\tau ; \theta ; \alpha_{c}^{m \tau}\right)=0 \\
& \mathscr{G}_{c}\left(S_{c} ; \theta ; \alpha_{c}^{m}\right)=\mathscr{G}_{c}^{\tau}\left(\tau ; \theta ; \alpha_{c}^{m \tau}\right)=\mathscr{K}_{c}
\end{aligned}
$$
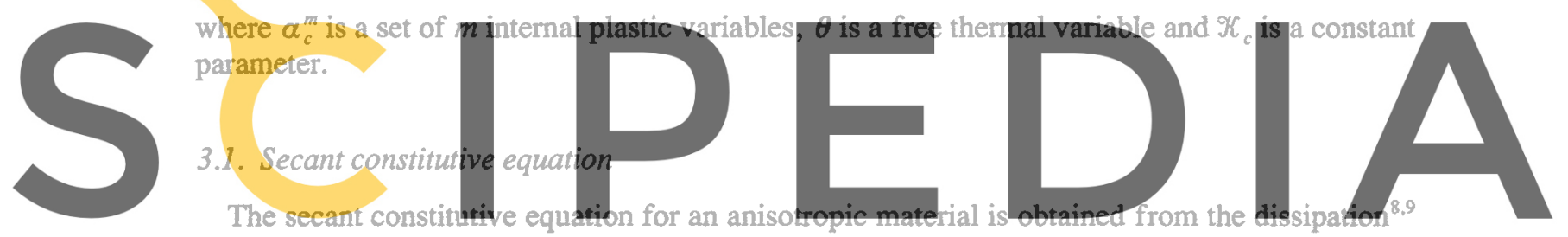

Register for free at https//www.scipedia.com to download the version without the watermark

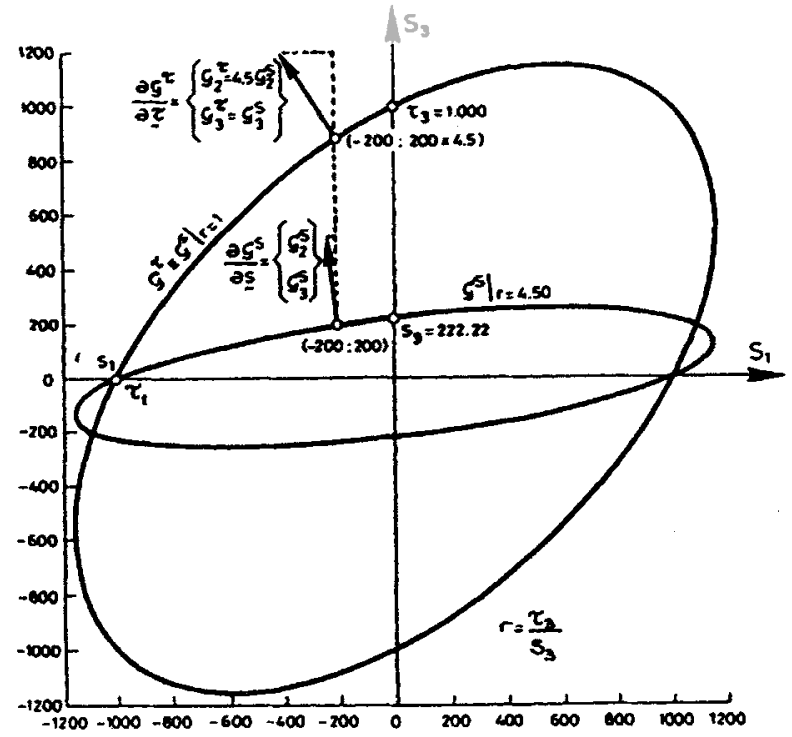

Figure 2. Differences in the plastic flow on the real $(r=1)$ and fictitious spaces $(r=1)$ and fictitious spaces $(r=4-5)$ 
occurring in a thermo-elastoplastic process in the real anisotropic space: ${ }^{4}$

$$
\begin{aligned}
\boldsymbol{S}_{c} & =m_{c}^{o} \frac{\partial \Psi_{c}\left(\boldsymbol{E}_{c}^{e} ; \theta ; \alpha_{c}^{m}\right)}{\partial \boldsymbol{E}_{c}^{e}} \\
& =\mathscr{T}_{c}^{-1}: \mathscr{\zeta}_{c}^{\tau}(\theta): \boldsymbol{\varepsilon}_{c}^{e}=\mathscr{T}^{-1}: \tau
\end{aligned}
$$

where $m_{c}^{o}$ is the density of the 'cth' compounding in the referential configuration, $\tau_{c}$ are the stresses in the fictitious isotropic space and $\Psi_{c}$ is the free energy of material formulated in the referential configuration under real stress state or on a fictitious one $\Psi_{c}^{r}{ }^{4}$

\subsection{Evolution law for the internal variables}

\section{From the transformation rules expressed by (4) and (5) and the definition of the plastic} potential function in the fictitious isotropic space, equation (6), the flow rule and the evolution of the internal plastic variables $\alpha_{c}^{m}$ are obtained as:
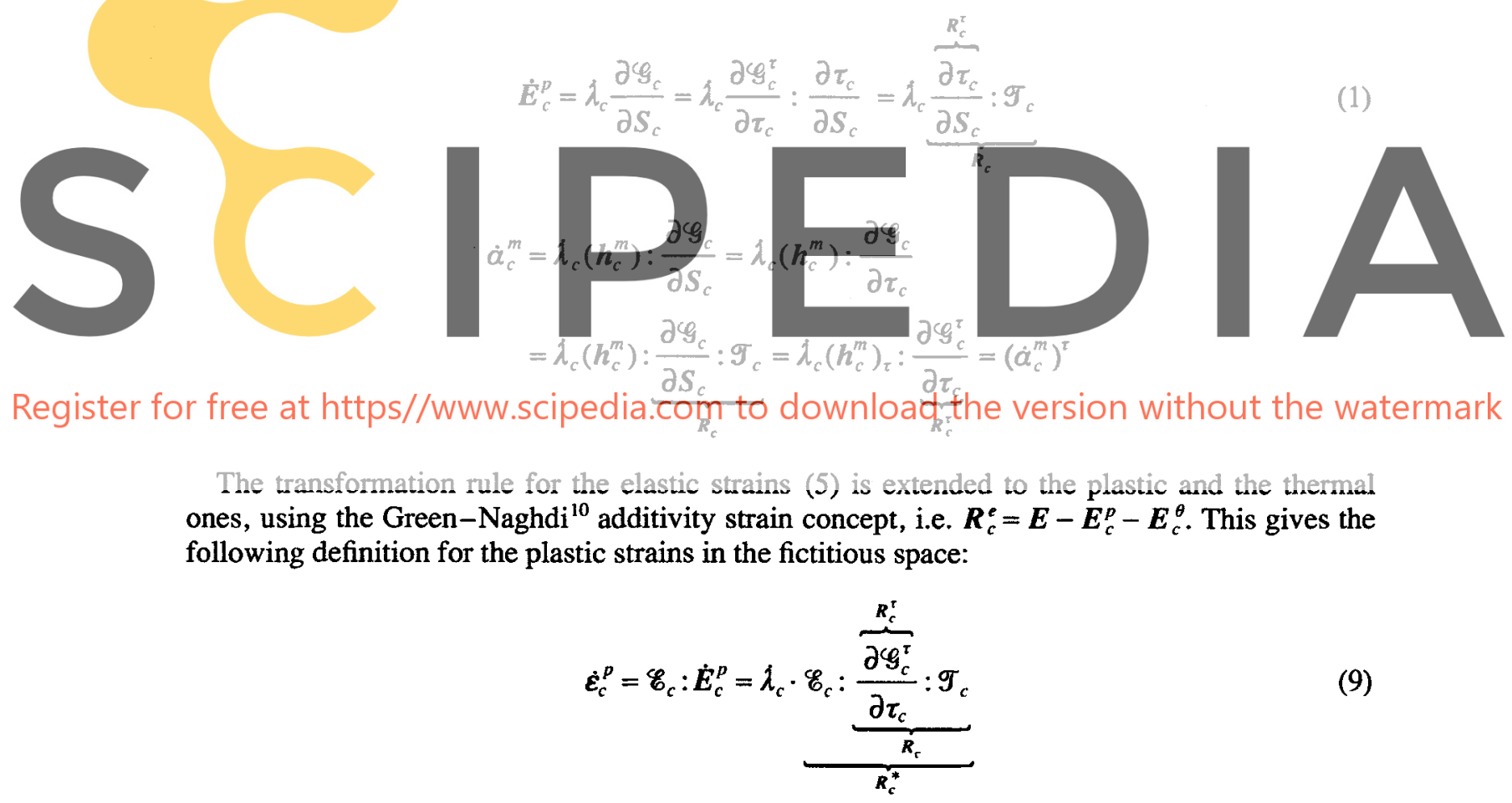

Register for free at https//www.scipedia.cop to downloa ${ }_{R_{c}} \tau_{c}$ the version without the wat
The transformation rule for the elastic strains (5) is extended to the plastic and the thermal ones, using the Green-Naghdi ${ }^{10}$ additivity strain concept, i.e. $\boldsymbol{R}_{c}^{e}=\boldsymbol{E}-\boldsymbol{E}_{c}^{p}-\boldsymbol{E}_{c}^{\theta}$. This gives the following definition for the plastic strains in the fictitious space:

$$
\dot{\boldsymbol{E}}_{c}^{p}=\mathscr{E}_{c}: \dot{\boldsymbol{E}}_{c}^{p}=\dot{\lambda}_{c} \cdot \mathscr{E}_{c}: \frac{\overbrace{\boldsymbol{E}_{c}^{\tau}}^{\frac{R_{c}^{\tau}}{\partial \mathscr{G}_{c}^{\tau}}}: \mathscr{T}_{c}}{\frac{\partial \tau_{c}}{\boldsymbol{R}_{\mathrm{c}}}}
$$

where $\left(\boldsymbol{h}_{c}^{m}\right)$ and $\left(\boldsymbol{h}_{c}^{m}\right)_{\tau}$ are tensorial functions to be determined ${ }^{6}$ for each of the $m$ internal variables involved, $\boldsymbol{R}_{c}^{\tau}$ is the plastic flow in the fictitious stress space, as shown in Figure 2, $\boldsymbol{R}_{c}$ is the plastic flow in the real stress space, $\boldsymbol{R}_{c}^{*}$ is the plastic flow in the fictitious isotropic stress space and $\hat{\boldsymbol{E}}_{c}^{p}$ is the fictitious plastic strain.

\subsection{Tangent constitutive equation}

The rate form of the constitutive equation is obtained from the temporal derivative of the 
constitutive secant expression (7) as

$$
\begin{aligned}
& \dot{S}_{c}=\frac{\partial S_{c}}{\partial E_{c}^{\mathrm{e}}}: \dot{\boldsymbol{E}}_{c}^{\mathrm{e}}+\frac{\partial \boldsymbol{S}_{c}}{\partial \theta} \dot{\boldsymbol{\theta}}
\end{aligned}
$$

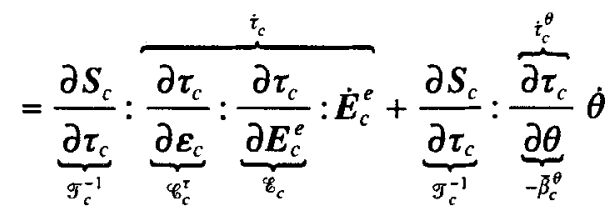

$$
\begin{aligned}
& =\mathscr{T}_{c}^{-1}: \underbrace{\mathscr{C}_{c}^{\tau}:\left(\dot{\varepsilon}-\dot{\varepsilon}_{c}^{p}\right)}_{\dot{i}_{c}}-\mathscr{T}_{c}^{-1}: \underbrace{\bar{\beta}_{c}^{\theta} \dot{\theta}}_{\dot{t}_{c}^{\theta}=\mathscr{\varphi}_{c}^{* \theta}: \dot{\varepsilon}_{c}^{\theta}}
\end{aligned}
$$

where $\overline{\boldsymbol{\beta}}_{c}^{\theta}=-\partial \tau_{c} / \partial \theta$ is the complementary thermal expansion coefficient (see Reference 8), and $\dot{\varepsilon}_{c}^{\theta}=\boldsymbol{a}_{c}^{\theta} \dot{\theta}$ is the thermal strain.

The plastic consistency condition applied in the fictitious space of the $c$ th compounding leads to the standard rate form of the constitutive equation in the fictitious isotropic space as

$$
t_{c}=\mathscr{C}_{c}^{\tau e p}: \dot{\varepsilon}-\mathscr{C}_{c}^{\tau \theta}: \dot{\varepsilon}_{c}^{\theta}
$$

where the tangent constitutive tensors are defined as:
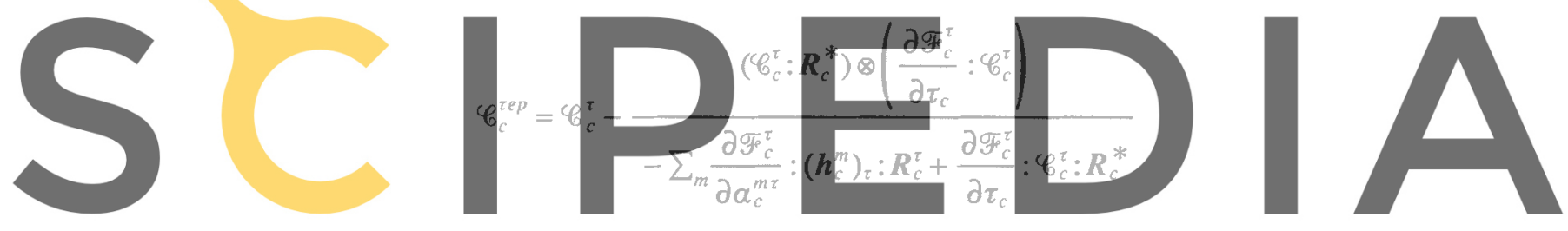

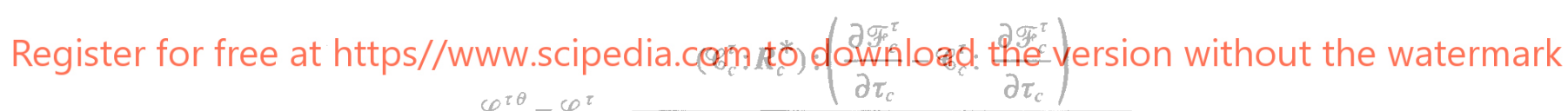

$$
\mathscr{C}_{c}^{\tau \theta}=\mathscr{C}_{c}^{\tau}-\frac{\left(\frac{\partial \tau_{c}}{\partial \tau_{c}}\right)}{-\sum_{m} \frac{\partial \mathscr{F}_{c}^{\tau}}{\partial \boldsymbol{\alpha}_{c}^{m \tau}}:\left(\boldsymbol{h}_{c}^{m}\right)_{\tau}: \boldsymbol{R}_{c}^{\tau}+\frac{\partial \mathscr{F}_{c}^{\tau}}{\partial \boldsymbol{\tau}_{c}}: \mathscr{C}_{c}^{\tau}: \boldsymbol{R}_{\boldsymbol{c}}^{*}}
$$

Substituting (11) into (10) gives the final expression of the rate constitutive equation in the real anisotropic compounding as:

$$
\begin{aligned}
\dot{S}_{c} & =\mathscr{T}_{c}^{-1}: \dot{t}=\mathscr{T}_{c}^{-1}:\left[\mathscr{C}_{c}^{\tau e p}: \dot{\boldsymbol{\varepsilon}}-\mathscr{C}_{c}^{\tau \theta}: \dot{\boldsymbol{E}}_{c}^{\theta}\right] \\
& =\underbrace{\mathscr{T}_{c}^{-1}: \mathscr{C}_{c}^{\tau e p}: \mathscr{E}_{c}}_{\mathscr{C}_{c}^{e p}}: \dot{\boldsymbol{E}}-\underbrace{\mathscr{T}_{c}^{-1}: \mathscr{C}_{c}^{\tau \theta}: \mathscr{E}_{c}}_{\mathscr{C}_{c}^{\theta}}: \dot{\boldsymbol{E}}_{c}^{\theta}
\end{aligned}
$$

Box 1. Non-linear finite element solution scheme for the anisotropic multiphase composite model.

1. Define the constitutive matrix, the participation of the different phases and the space transformation tensors:

$$
\begin{aligned}
& \mathbf{f}_{c}^{\tau}, \mathbf{f}_{e} \mathscr{C}_{c}^{\tau}, \mathscr{C}_{c} \\
& \mathscr{T}_{c}=\mathbf{f}_{c}^{\tau} \otimes \mathbf{f}_{c}^{-1} \\
& \mathscr{E}_{c}=\mathscr{C}_{c}^{\tau-1}: \mathscr{T}_{c}: \mathscr{C}_{c}
\end{aligned}
$$




$$
\begin{aligned}
& { }^{1}\left(\mathscr{C}_{c}^{r e p}\right)^{o}=\mathscr{C}_{c}^{\tau} \\
& { }^{1}\left(\mathscr{C}_{c}^{e p}\right)^{o}=\mathscr{T}_{c}^{-1}:{ }^{1}\left(\mathscr{C}_{c}^{\tau e p}\right)^{o}: \mathscr{C}_{c} \rightarrow{ }^{1}\left(\mathscr{C}^{e p}\right)^{o}=\sum_{c} k_{c}{ }^{1}\left(\mathscr{C}_{c}^{e p}\right)^{o} \\
& { }^{1}\left(\mathscr{C}_{c}^{\tau \theta}\right)^{o}=0 \rightarrow{ }^{1}\left(\mathscr{C}^{\theta}\right)^{o}=\sum_{c} k_{c}{ }^{1}\left(\mathscr{C}_{c}^{\tau \theta}\right)^{o}=0
\end{aligned}
$$

$\begin{array}{ll}\Rightarrow \text { LOOP OVER LOAD INCREMENTS } & : \text { nth increment } \\ \Rightarrow \text { LOOP OVER CONVERGENCE ITERATIONS } & : \text { ith iteration }\end{array}$

2. Compute the tangent stiffness matrix for each element and the structure:

$$
\begin{aligned}
{ }^{n}\left(\boldsymbol{K}^{(e)}\right)^{i-1} & =\int_{V} \boldsymbol{B}:^{n}\left(\mathscr{C}^{e p}-\mathscr{C}^{\theta}\right)^{i-1}: \boldsymbol{B} \mathbf{d} V \\
{ }^{n}(\boldsymbol{K})^{i-1} & =\mathbb{A}_{e=1}^{n e !}\left(\boldsymbol{K}^{(e)}\right)^{i-1} .
\end{aligned}
$$

3. Compute the nodal displacements increments and the strains in the composite:
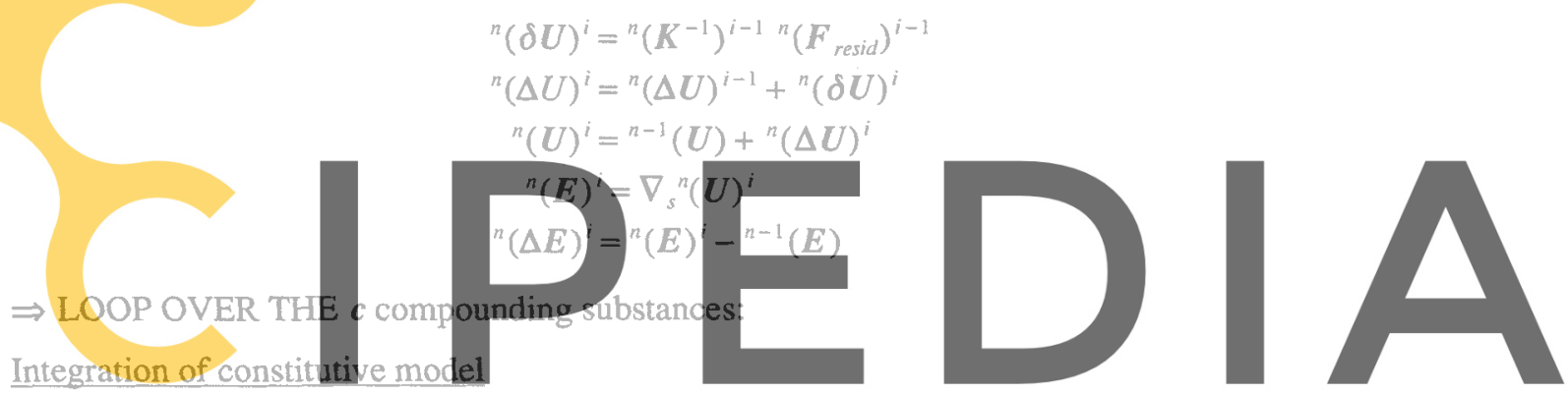

4. Compute the predicted stress for each isotropic plastic compound: Register for free at https//www.scipedia.com to download the version without the watermark ${ }^{n}\left(\tau_{c}^{*}\right)^{i}=\left(\mathscr{C}_{c}{ }^{\tau}\right):\left[\mathscr{E}_{c}:{ }^{n}(\mathbb{E})^{i}\right]-\mathscr{C}_{c}^{\tau}:\left[{ }^{n-1}\left(\varepsilon_{c}^{p}\right)\right]-\left({ }^{n} \theta^{i}-{ }^{n} \theta^{i-1}\right) \bar{\beta}_{c}^{\theta}$

5. Integrate the plastic constitutive equation for each compound (Euler backward scheme):

$\Rightarrow$ LOOP OVER INNER CONVERGENCE ITERATIONS: $k$ th iteration:

$$
\begin{gathered}
\text { for } k=1:{ }^{n}\left(\tau_{c}\right)^{i, 0}={ }^{n}\left(\tau_{c}^{*}\right)^{i},{ }^{n}\left(\Delta \varepsilon_{c}{ }^{p}\right)^{i, 0}=0 \\
\text { * }{ }^{n}\left(\tau_{c}\right)^{i, k}={ }^{n}\left(\tau_{c}\right)^{i, k-1}-\left(\mathscr{C}_{c}^{\tau}\right):{ }^{n}\left(\Delta \varepsilon_{c}{ }^{p}\right)^{i, k-1}
\end{gathered}
$$

Is : $\mathscr{F}_{c}^{r}\left(\tau_{c}, \theta, \alpha_{c}^{m}\right)_{\tau_{c}^{i, k-1}} \leqslant 0 \Rightarrow$ (no yielding) GOTO 10

(yielding)

$$
\begin{aligned}
{ }^{n}\left(\Delta \varepsilon_{c}^{p}\right)^{i, k} & =\left(\Delta \lambda_{c}\right)^{k} \cdot \mathscr{E}_{c}:^{n}\left(\frac{\partial \mathscr{G}_{c}^{\tau}}{\partial \tau_{c}}\right)_{n_{r_{c}^{i, k}}^{k}}^{k}: \mathscr{I}_{c} \\
{ }^{n}\left(\boldsymbol{\varepsilon}_{c}^{p}\right)^{i, k} & ={ }^{n}\left(\boldsymbol{\varepsilon}_{c}^{p}\right)^{i, k-1}={ }^{n}\left(\Delta \boldsymbol{\varepsilon}_{c}^{p}\right)^{i, k} \\
{ }^{n}\left(\Delta \boldsymbol{\alpha}_{c}{ }^{m}\right)^{i, k} & =\left(\Delta \lambda_{c}\right)^{k n}\left(\boldsymbol{h}_{c}^{m}\right)^{i, k} ;{ }^{n}\left(\Delta \boldsymbol{\varepsilon}_{c}^{p}\right)^{i, k} \\
{ }^{n}\left(\alpha_{c}^{m}\right)^{i, k} & ={ }^{n}\left(\alpha_{c}{ }^{m}\right)^{i, k-1}+{ }^{n}\left(\Delta \alpha_{c}^{m}\right)^{i, k} \\
k & =k+1 \text { Go back to \&\& }
\end{aligned}
$$




\section{Compute the plastic tangent constitutive matrix for each phase:}

$$
\begin{aligned}
& { }^{n}\left(\mathscr{C}_{c}^{\tau e p}\right)^{i}=\left\{\mathscr{C}_{c}^{\tau}-\frac{\left(\mathscr{C}_{c}^{\tau}: \boldsymbol{R}_{c}^{*}\right) \otimes\left(\frac{\partial \mathscr{F}_{c}^{\tau}}{\partial \tau_{c}}: \mathscr{C}_{c}^{\tau}\right)}{-\sum_{m} \frac{\partial \mathscr{F}_{c}^{\tau}}{\partial \alpha_{c}^{m \tau}}\left(\boldsymbol{h}_{c}^{m}\right): \boldsymbol{R}_{c}^{\tau}+\frac{\partial \mathscr{F}_{c}^{\tau}}{\partial \tau_{c}}: \mathscr{C}_{c}^{\tau}: \boldsymbol{R}_{c}^{*}}\right\}_{n_{\tau} i} \\
& { }^{n}\left(\mathscr{G}_{c}^{\tau \theta}\right)^{i}=\left\{\mathscr{C}_{c}^{\tau}-\frac{\left(\mathscr{G}_{c}^{\tau}: \boldsymbol{R}_{c}^{*}\right) \otimes\left(\frac{\partial \mathscr{F}_{c}^{\tau}}{\partial \tau_{c}}-\mathscr{C}_{c}^{\tau}: \frac{\partial \mathscr{F}_{c}^{\tau}}{\partial \tau_{c}}\right)}{-\sum_{m} \frac{\partial \mathscr{F}_{c}^{\tau}}{\partial \alpha_{c}^{m \tau}}\left(\boldsymbol{h}_{c}^{m}\right): \boldsymbol{R}_{c}^{\tau}+\frac{\partial \mathscr{F}_{c}^{\tau}}{\partial \tau_{c}}: \mathscr{C}_{c}^{\tau}: \boldsymbol{R}_{c}^{*}}\right\} \\
& c=c+1 \text { Go back to }
\end{aligned}
$$

\section{Compute:}

$$
\begin{aligned}
n\left(S_{c}\right)^{i} & =\mathscr{T}_{c}^{-1}{ }^{n}\left(\tau_{c}\right)^{i} \\
{ }^{n}\left(\mathscr{C}_{c}^{e p}\right)^{i} & =\mathscr{T}_{c}^{-1}:{ }^{n}\left(\mathscr{C}_{c}^{r e p}\right)^{i}: \mathscr{E}_{c} \\
{ }^{n}\left(\mathscr{C}_{c}^{\theta}\right)^{i} & =\mathscr{T}_{c}^{-1}:{ }^{n}\left(\mathscr{C}_{c}^{r \theta}\right)^{i}: \mathscr{E}_{c}
\end{aligned}
$$
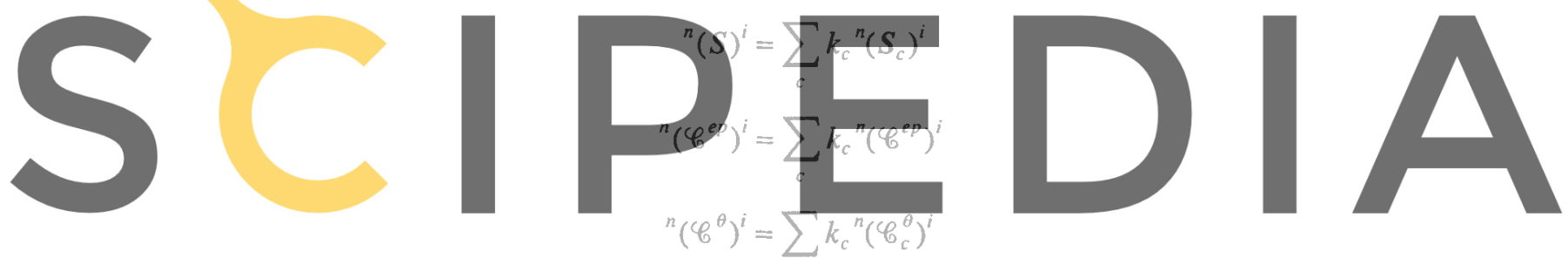

Register for free at https//www.scipedia.com to ${ }^{c}$ download the version without the watermark

8. Compute the residual force vector and check convergence:

$$
\begin{gathered}
{ }^{n}\left(\boldsymbol{F}_{\text {resid }}^{(e)}\right)^{i}=\int_{V} \boldsymbol{B}:{ }^{n}(\boldsymbol{S})^{i} \mathrm{~d} V-\boldsymbol{F}_{\text {ext }} \\
{ }_{n}\left(\boldsymbol{F}_{\text {resid }}\right)^{i}=\mathbf{A}_{e=1}^{n e l}{ }^{n}\left(\boldsymbol{F}_{\text {resid }}^{(e)}\right)^{i} \\
I s\left\|\boldsymbol{F}_{\text {resid }}\right\|>\boldsymbol{\|}\left\|\boldsymbol{F}_{\text {ext }}\right\| ? \Rightarrow i=i+1 . \text { Go back to } 2 \\
\text { STOP }
\end{gathered}
$$

\section{EXAMPLES}

\subsection{Analysis of a fibre reinforced composite}

In this example, a plane rectangular specimen of a fibre reinforced composite material ${ }^{4}$ is analysed. Figure 3 shows the specimen geometry, the boundary conditions and the finite element mesh of the sixteen 4-node quadrilateral elements used. A prescribed longitudinal displacement has been imposed at both ends of the specimen as shown in Figure 3.

The specimen is composed of $80 \%$ of isotropic matrix and $20 \%$ of long fibres. The fibres are considered aligned along the longitudinal direction in the first test, and then are placed along the transverse direction. 

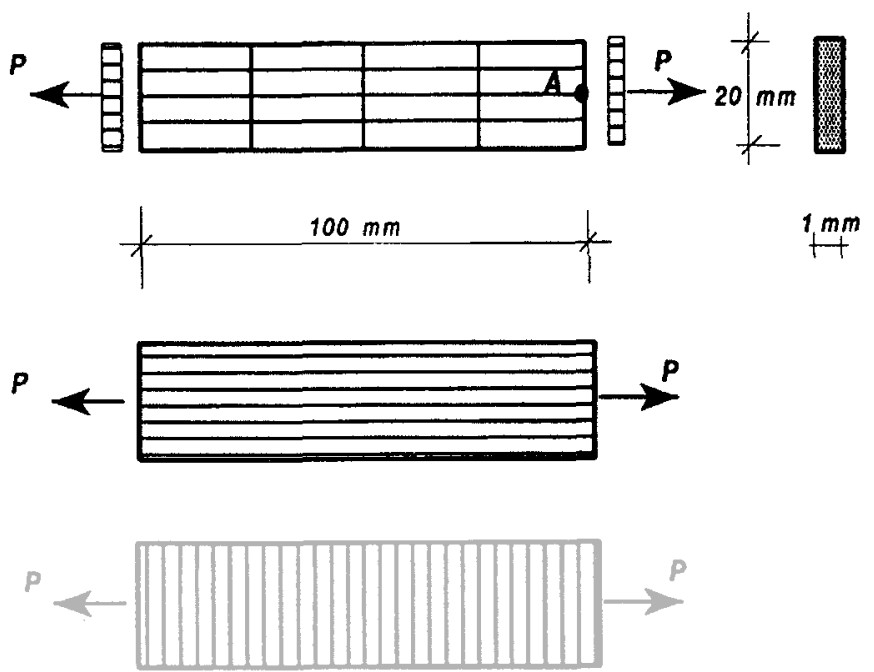

Figure 3. Fibre reinforced specimen. Geometry, boundary conditionn, loading and finite element mesh

Material properties are: Matrix isotropic material: $E=7.24 \mathrm{~m} / \mathrm{mm}^{2}, v=0.33$, limit elastic strength $f^{\tau}=0.036 \mathrm{tn} / \mathrm{mm}^{2}$ and $80 \%$ of fraction volume participation. In this case isotropic associated Von-Mises plasticity has been assuned. Fibres anisotropic model: $E=84.4 \mathrm{tn} / \mathrm{mm}^{2}$ and limit elastic strength $f^{\tau}=0.2283 \mathrm{~m} / \mathrm{mm}^{2} ; 20 \%$ of fraction volume participation used. Von-Mises yield function and plastic flow along the fibre direction in the isotropic space has been assuned. Perfect plastic behaviour for both materials has beer a

Figure 4(1) displays the stress-strain behaviour for the conposite with longitudinal fbres, showing the behaviour of the composite (curve $b$ ), the matrix (curve $c$ ) and the fibres (curve $a$ ).

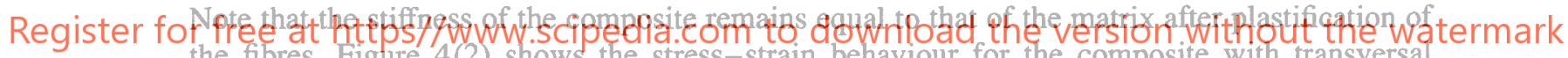
the fibres. Figure 4(2) shows the stress-strain behaviour for the composite with transversal fibres. Curve $(a)$ shows the evolution of the longitudinal stress in the composite; curve (b)
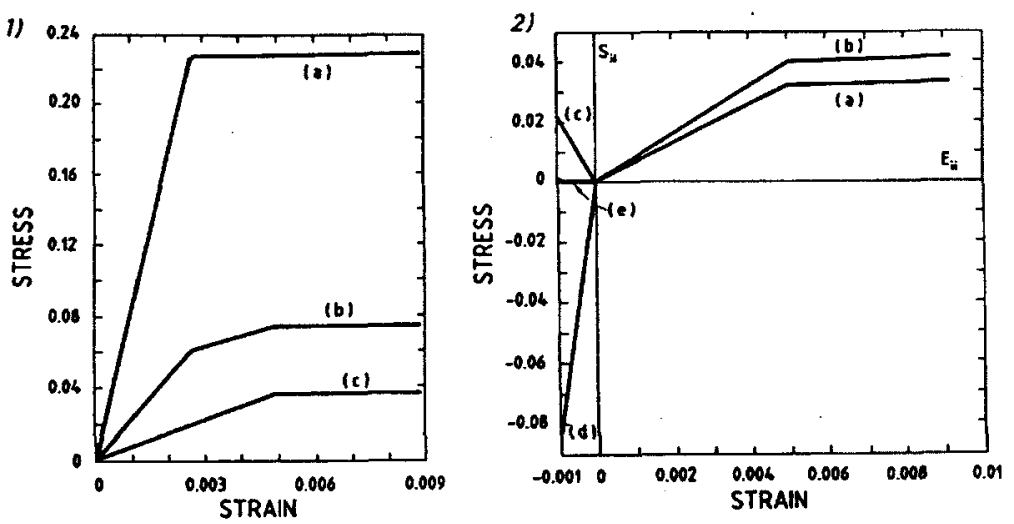

Figure 4. Fibre reinforced composites: Stress-strain curves in the centre of the specimen. Case (1): Longtitudinal fibres. Behaviour on the longitudinal direction of the fibres (curve $a$ ), composite (curve $b$ ) and matrix (curve $c$ ). Case (2): Transverse fibres. Behaviour on the longitudinal direction of the composite (curve $a$ ) and matrix (curve $b$ ). Behaviour on the transverse direction of the matrix (curve $c$ ), fibres (curve $d$ ) and composite (curve $e$ ) 
shows that the strength of the matrix is greater than that of the composite. This can be explained by the small contribution of the transverse fibres to the global longitudinal stiffness. Therefore, the resisting material has $20 \%$ less transverse cross area with the corresponding reduction in stiffness. Curve $(c)$ in the same Figure shows the transversal tension stress in the matrix and the corresponding compression stress in the fibres (curve $d$ ).

Finally, curve $(e)$ shows the null 'global' strength of the composite in the transverse direction, as expected.

Figure 5 shows the load-displacement curves for the two fibre orientations studied.

The numerical results obtained are in good agreement with the expected values, thus showing the ability of the model proposed to analyse fibre reinforced composites.

\subsection{Test on steel reinforced concrete treated as a fibre-matrix composite material}

The proposed model has also been tested in the analysis of a simple concrete specimen subjected to axial loading as shown in Figure 6. Plane stress conditions have been assumed. Three kinds of composite material are considered:

Composite (a): 100\% of concrete. Plastic fracture behaviour, and Mohr-Coulomb yield and potential functions are assumed.

Composite $(b): 90 \%$ of concrete $+10 \%$ of steel longitudinal bars. For concrete: plastic fracture behaviour and Mohr-Coulomb yield and potential functions are assumed. Elastic behaviour has been assumed for the steo bar.

Composite (c): $90 \%$ and Mohr-Coulomb

Mises plastic behaviou

Additional properties for the concrete are: Young's modulus coefficient $v=0.24$, friction and dilatancy
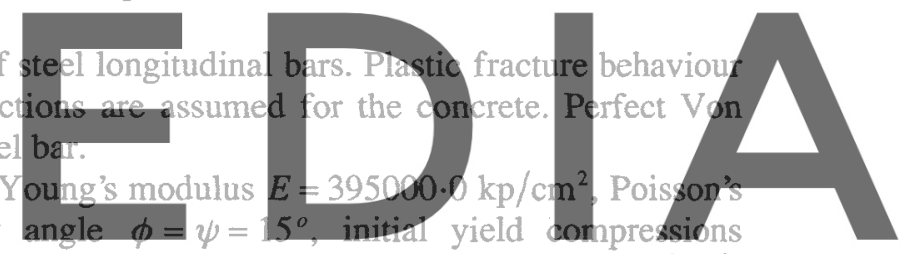
strength $f_{C}^{\tau}=229.0 \mathrm{kp} / \mathrm{cm}^{2}$, maximum level of compression strength $f_{p k}^{\tau}=328.0 \mathrm{kp} / \mathrm{cm}^{2}$,

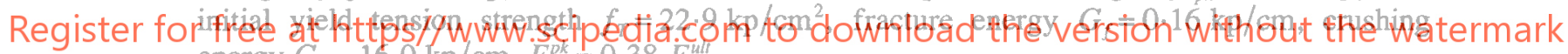
energy $G_{c}=16.0 \mathrm{kp} / \mathrm{cm}, E^{p k}=0.38 E^{\text {ult }}$.

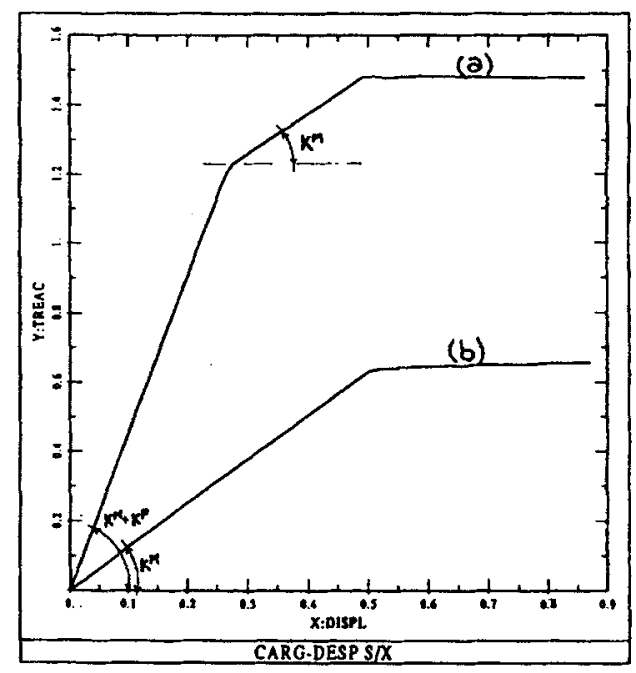

Figure 5. Load-displacement curves in point ' $\mathrm{A}$ ' of the speciment: $(a)$ longitudinal fibres, $(b)$ transverse fibres 


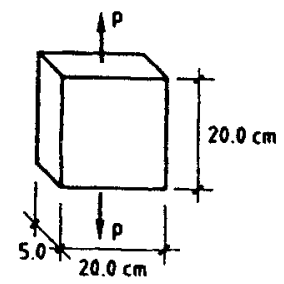

Concrete-Steel composite specinen. $90 \%$ of concrete (fracture model] $10 \%$ of longitudinal steal bars

(elasto-plastic nodet)

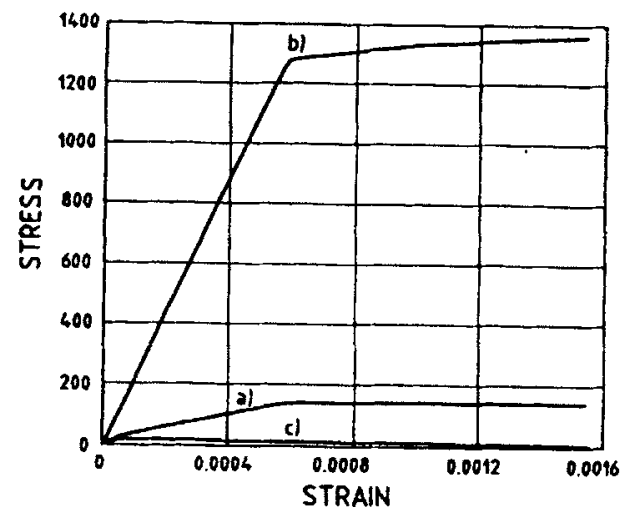

Figure 6. Steel reinforced concrete specimen: geometry, loading condition and stress-strain behaviour for each compounding of the concrete composite c: curve $(a)$ represents the composite behaviour and curves $(b)$ represents the composite behaviour and curves $(b)$ and $(c)$ the steel and concrete components
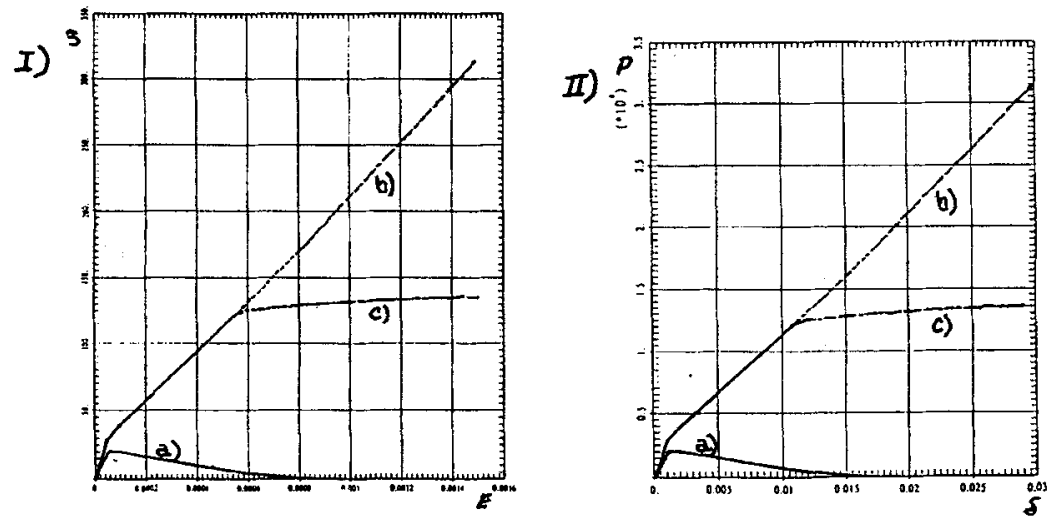

Figure 7. Case I: Stress-strain curves for concrete composites $(a),(b)$ and (c); case II: load-displacements curves 
Additional properties for the steel bars are: $E=2100000 \cdot 0 \mathrm{kp} / \mathrm{cm}^{2}, v=0 \cdot 30, \phi=\psi=15^{\circ}$, $f_{C}=f_{T}=1200 \cdot 0 \mathrm{kp} / \mathrm{cm}^{2}$. A tension load has been applied as shown in Figure 6 . This Figure shows the stress-strain behaviour for each compounding of the composite $c$.

The apparent small hardening exhibited by the steel is due to the transversal interaction between concrete and steel. The uniaxial steel strength $\left(1200 \cdot 0 \mathrm{kp} / \mathrm{cm}^{2}\right)$ is reached when the concrete response vanishes.

Figures 7 (a) and (b), respectively show the stress-strain and load-displacement curves for the composites (a), (b) and (c). The plastification points were verified by means of a simple theory. Full agreement with the present model was obtained.

\section{CONCLUDING REMARKS.}

The theoretical framework presented here combines the basic concepts from multiphase theory with those of anisotropic mapped theory. Such a framework provides a powerful tool for modeling the behaviour of several composite materials. Moreover, the formulation presented can simulate the non-linear behaviour of each component as well as the global fracture behaviour for the composite material. Also, induced anisotropy is intrinsic to the formulation of the constitutive law for the compounding. This will allow us to model failure by strain localisation, typical of softening materials.

\section{REFERENCES}

1. Trusdell, C. and Toupin, R., 'The classical field theories', in S. Flugge (Ed.), Handbuch der Physik $I I I / I$, Springer Verlag, Berlin, 1960.

2. Betten, J., 'Creep theory of anisotropic solids', J. Rheol., 25, (6), 565-581 (1981).

3. Betten, J., "Application of tensor functions to the formulation of yield criteria for anisotropic materials', Int. J. Plast., 4, 29-46 (1988).

4. Oller, S., Botello, S., Miquel, J. and Oñate, E., 'An anisotropic elasto-plastic model based on an isotropic formulation', Int. J. Eng. Comput., 12, 245-262 (1995).

5. Oller, S., Botello, S., Miquel, J. and Oñate, E., 'A multiphase constitutive model for composite materials', Int. J. Solids Struct, 33, (17), 2501-2518 (1996).

6. Lubliner, J., Oliver, J., Oller, S., Oñate, E., 'A plastic damage model for non linear analysis of concrete', Int. J. Solids Struct., 25, (3), 299-326 (1989).

7. Oller, S., Oñate, E., Oliver, J., Lubliner, J., 'Finite element non-linear analysis of concrete structures using a plastic-damage model', Eng. Fract. Mech., 35, (1/2/3), 219-231 (1990).

8. Lubliner, J., Plasticity Theory, Macmillan Publishing, U.S.A., 1990.

9. Malvern, L., 'Introduction to the mechanics of continuous medium', Prentice Hall, U.S.A., 1969

10. Green A. Naghdi, P., 'A general theory of an elastic-plastic continua', Arch. Ration. Mech. Anal., 18, 19-281 (1964). 